\section{Cureus}

\title{
Shining a Light on Overnight Education: Hospitalist and Resident Impressions of the Current State, Barriers, and Methods for Improvement
}

\author{
Sara N. Sani ${ }^{1}$, Emily Wistar ${ }^{1}$, Lien Le ${ }^{2}$, David Chia ${ }^{1}$, Lawrence A. Haber ${ }^{1}$ \\ 1. Medicine, University of California San Francisco, San Francisco, USA 2. Gastroenterology, University of California \\ San Diego, San Diego, USA
}

$\square$ Corresponding author: Lawrence A. Haber, lawrence.haber@ucsf.edu Disclosures can be found in Additional Information at the end of the article

\begin{abstract}
Introduction: Restrictions on resident work hours and increased requirements for resident supervision have led to night float rotations overseen by overnight hospitalists (nocturnists). The educational value of night float rotations for residents has traditionally been low and studies have yet to elucidate the optimal role of nocturnists in resident education.
\end{abstract}

Methods: We performed a cross-sectional survey of all residents within our training program and attending hospitalists in the department of medicine at our three teaching medical centers. Questions sought to investigate the current state of overnight education within an internal medicine residency program, understand barriers to overnight education, and define best practices for nighttime teaching.

Results: Both attending and resident physicians reported low satisfaction with the current state of overnight education, while simultaneously expressing high levels of interest in performing and receiving dedicated nighttime teaching. Attending and resident physicians identified similar barriers to overnight teaching (clinical work, time coordination, provider fatigue) and agreed on the ideal format for overnight didactics (case-based, chalk-talk, 20-minute duration between $10 \mathrm{pm}$ to $2 \mathrm{am}$ ).

Conclusion: Our study identifies a desire by both faculty and trainees for increased overnight teaching and offers a simple initial framework for programs to improve overnight housestaff education utilizing nocturnist providers.

Received 06/08/2018

Review began 06/19/2018

Review ended 07/03/2018

Published 07/06/2018

C) Copyright 2018

Sani et al. This is an open access article distributed under the terms of the

Creative Commons Attribution License CC-BY 3.0., which permits unrestricted use, distribution, and reproduction in any medium, provided the original author and source are credited.
Categories: Internal Medicine, Medical Education

Keywords: overnight education, nocturnist, nightfloat, resident curriculum

\section{Introduction}

Increasing resident supervision mandates and work hour restrictions, enacted in response to the 2011 Accreditation Council for Graduate Medical Education (ACGME) regulations [1], have led to the prevalence of overnight hospitalists (nocturnists) in training environments [2]. While studies have shown nocturnists to be a key resource for improving housestaff well-being and patient care oversight [3-4], their role in formal overnight housestaff education has not been well studied.

Studies demonstrate negative housestaff perceptions of the educational value of night float rotations [56]. Recent investigation into improving overnight trainee education has focused on formalizing residentled curricula [7-8], without considering the role nocturnists can play in enhancing nighttime learning. This is despite evidence that residents find increased educational value to nighttime rotations when working alongside overnight faculty [9-10].

We sought to investigate the current state of overnight resident education at our tri-institutional training program, understand barriers to nighttime teaching, and elicit best practices to guide programs

How to cite this article

Sani S N, Wistar E, Le L, et al. (July 06, 2018) Shining a Light on Overnight Education: Hospitalist and Resident Impressions of the Current State, Barriers, and Methods for Improvement. Cureus 10(7): e2939. DOI 10.7759/cureus.2939 
developing overnight resident curriculum utilizing nocturnist providers.

\section{Materials And Methods}

The study was conducted at a tertiary care medical center, public safety-net hospital, and Veteran Affairs hospital, all associated with the University of California, San Francisco Internal Medicine Residency Program. There are 186 residents in the training program. First-year residents work a minimum of one week of night float consisting of overnight cross-coverage of patients admitted to the general medicine service. Second and third-year residents complete four weeks on night float rotations, assisting interns with cross-coverage and admitting general medicine patients.

At each hospital, there is a minimum of one nocturnist present every evening. The nocturnist staffs overnight admissions to the intensive care unit, discusses newly admitted step-down patients, and provides supervision of care for any patients undergoing a major change in clinical status. The nocturnist also covers direct care services, performs medicine consults, and admits patients in rotation with housestaff. Within the training program, no overnight educational curriculum or explicit instruction for overnight teaching currently exists.

Three full-time nocturnists, one from each of our three medical centers, developed survey content based on study goals, available literature, and personal experience. Surveys were piloted on a select number of residents and faculty for content and ease of use, with questions further refined in works in progress meetings.

We e-mailed surveys to all residents and attending hospitalists at each training site. We excluded residents who had not worked a night shift in the past year and hospitalists who had not worked a night shift in the past five years. Informed consent was embedded within the e-mail survey request. We did not collect unique identifiers from respondents and no incentive was offered for completion of the survey. Surveys were composed of Likert-style questions as well as free text responses. Survey data were anonymous and downloaded to a database (Qualtrics, Provo, UT) by a third party. Statistical tests of significance were performed using Fisher's exact test and t-test. The study was approved by the medical center's Institutional Review Board.

\section{Results}

\section{Respondents}

There were 64 hospitalist and 42 resident survey respondents (response rate of 34\% and 23\%, respectively). 19 hospitalists were excluded based on study criteria. Nocturnist shifts were primarily staffed by junior faculty with a mean of 3.3 years of attending experience (range $0-13$ years), performing 30.7 shifts per academic year (range 1-110 shifts). All years of medicine residency training were represented (PGY-1 36\%, PGY-2 45\%, PGY-3 21\%).

\section{Current state and barriers}

Both attending and resident physicians reported low levels of satisfaction with the current state of overnight education ( $24 \%$ and $12 \%$ respectively, $p=0.17$ ), yet high levels of interest in performing and receiving dedicated nighttime teaching ( $67 \%$ and $78 \%, \mathrm{p}=0.34)$. We found a misperception of disinterest between the groups with $68 \%$ of hospitalists reporting perceived resident disinterest in receiving overnight teaching, while only $33 \%$ of residents self-reported disinterest $(\mathrm{p}=0.005)$. Similarly, $71 \%$ of residents reported perceived attending disinterest in delivering teaching, while only $29 \%$ of hospitalists self-reported disinterest in delivering teaching $(\mathrm{p}<0.001)$. Attendings and residents identified similar barriers to overnight teaching including clinical work ( $84 \%$ and $86 \%$, respectively), difficulty coordinating meeting time ( $76 \%$ and $71 \%$ ), and provider fatigue (40\% and 54\%); there was no significant difference in responses between the two groups.

\section{Ideal overnight practices}

Residents responded that the factors that would most facilitate nighttime teaching were protecting housestaff from clinical duties (57\%), setting explicit expectations that teaching occur nightly (45\%), and assigning a designated time slot for teaching (36\%). Faculty responses were similar, except that 


\section{Cureus}

hospitalists and residents disagreed over the benefit of protecting trainees from clinical duties to facilitate teaching (33\% and $57 \%$ respectively, $\mathrm{p}=0.03$ ).

When queried about the ideal model for overnight discrete teaching sessions, hospitalist and resident respondents agreed that ideal overnight didactics should be case-based ( $78 \%$ and $63 \%$ ) chalk talks (58\% and 71\%) of approximately 20 minutes duration (18.4 and 21.5 minutes). Nearly all hospitalists and residents felt that overnight teaching should occur between the hours of 10:00 pm to 2:00 am. There were no statistically significant differences in responses between groups. The above findings, in addition to the six overlapping nighttime teaching topics that both groups reported as high-yield are summarized in Table 1 .

Structure
Format
Duration
Timing
High yield topic 1
High yield topic 2
High yield topic 3
High yield topic 4
High yield topic 5
High yield topic 6

Respondent Recommendations

Case-based chalk-talk

20 minutes

$10: 00 \mathrm{pm}-2: 00 \mathrm{am}$

Codes and rapid responses

Cross-cover and common night calls

Overnight emergencies

Hypoxia and respiratory distress

Sepsis and shock

Chest pain and myocardial infarction

TABLE 1: Recommended night curriculum structure

\section{Discussion}

Our study contributes to a limited body of literature on overnight resident education and is the first, to our knowledge, to investigate perspectives on the role of overnight hospitalists in nighttime didactics. Similar to prior studies, we found low satisfaction with current overnight education among both hospitalist and resident respondents. Our findings, however, dispel the belief that attending and resident physicians are disinterested in receiving or providing nighttime teaching.

In 2014, Hanson et al. called for the development of skilled nighttime educators to realize the unique educational potential of night shifts [11]. Our results speak to the potential of nocturnists in training environments to act as teaching attendings. Even with brief contact, formalizing nocturnist involvement in overnight didactics may present an opportunity for junior faculty to engage with trainees in an educational capacity, foster job satisfaction, and obtain teaching evaluations necessary for academic advancement. Accordingly, junior faculty who perform overnight shifts may benefit from additional teaching training and programs should consider teaching skills as a unique factor when evaluating nocturnist hires.

Our findings reinforce prior reports on barriers to maintaining overnight teaching, namely provider fatigue and completing clinical duties [12]. Clinical needs supersede didactics, especially at night when physician staffing may be reduced. This may account for resident and nocturnist perceptions of lack of interest in receiving and delivering overnight education. It may also be possible that a lack of clear expectations on the part of hospitalists or residents, around the importance of teaching at night or how best to incorporate education into often unstructured overnight clinical hours, may also lead to perceived disinterest. The simplest intervention proposed by our respondents to increase the frequency of night 
teaching is providing the clear expectation to all overnight providers that teaching will occur.

Our data present relatively granular recommendations for programs to build an overnight curriculum. We found agreement between both faculty and trainees that nighttime sessions should be brief, case-based, chalk talks, occurring between $10 \mathrm{pm}$ and $2 \mathrm{am}$. Our high-yield teaching topics overlap with prior literature [8], but have the added benefit of perspective from trainees at varying levels of seniority in addition to that of practicing overnight faculty.

There are several limitations to our study. First, the findings represent the experience of internal medicine housestaff within a single residency program and may not be reflective of other programs. The experience of faculty respondents does represent that of three distinct hospitals, though may not be representative of experiences outside of our academic program. Second, we had low response rates from trainees, though not dissimilar absolute numbers compared with similar studies [9]. Finally, we solicited perceptions and preferences of residents and faculty members with regard to current state, barriers, and ideal practices. Whether these preferences would be well received in practice has yet to be determined.

\section{Conclusions}

Our study underscores a desire for increased overnight teaching on the part of both learners and faculty. Future steps will focus on building overnight curricula delivered by nocturnists using evidence-based structure and topics, defining measures of success for overnight education, and examining teaching skills of overnight faculty.

\section{Additional Information \\ Disclosures}

Human subjects: All authors have confirmed that this study did not involve human participants or tissue. Animal subjects: All authors have confirmed that this study did not involve animal subjects or tissue. Conflicts of interest: In compliance with the ICMJE uniform disclosure form, all authors declare the following: Payment/services info: All authors have declared that no financial support was received from any organization for the submitted work. Financial relationships: All authors have declared that they have no financial relationships at present or within the previous three years with any organizations that might have an interest in the submitted work. Other relationships: All authors have declared that there are no other relationships or activities that could appear to have influenced the submitted work.

\section{References}

1. Accreditation council for graduate medical education. common program requirements . (2011). Accessed: June 13, 2018:

http://www.acgme.org/Portals/0/PFAssets/ProgramResources/Common_Program_Requirements_07012011.

2. Oshimura JM, Sperring J, Bauer BD, Carroll AE, Rauch DA: Changes in inpatient staffing following implementation of new residency work hours. J Hosp Med. 2014, 10:640-645. doi:10.1002/jhm.2242

3. Bolster L, Rourke L: The effect of restricting residents' duty hours on patient safety, resident well-being, and resident education: an updated systematic review. J Grad Med Educ. 2015, 7:349-363. doi:10.4300/JGME-D-14-00612.1

4. Haber LA, Lau CY, Sharpe BA, Arora VM, Farnan JM, Ranji SR: Effects of increased overnight supervision on resident education, decision-making, and autonomy. J Hosp Med. 2012, 7:606-610. 10.1002/jhm.1959

5. Luks AM, Smith CS, Robins L, Wipf JE: Resident perceptions of the educational value of night float rotations. Teach Learn Med. 2010, 22:196-201. 10.1080/10401334.2010.488203

6. Lefrak S, Miller S, Schirmer B, Sanfey H: The night float system: ensuring educational benefit . Am J Surg. 2005, 189:639-642. 10.1016/j.amjsurg.2004.11.034

7. Brady AK, O'Rourke P, Kobayashi T, et al.: A novel, resident-led curriculum for night float rotations . J Grad Med Educ. 2015, 7:289-90. 10.4300/JGME-D-14-00685.1

8. Golbus JR, Manly DA, Wonneberger KA, et al.: Implementation of a novel, resident-led, nocturnal curriculum. J Grad Med Educ. 2015, 7:417-421. 10.4300/JGME-D-14-00735.1

9. Trowbridge RL, Almeder L, Jacquet M, Fairfield KM: The effect of overnight in-house attending coverage on perceptions of care and education on a general medical service. J Grad Med Educ. 2010, 2:53-56. 10.4300/JGME-D-09-00056.1

10. Phy MP, Offord KP, Manning DM, Bundrick JB, Huddleston JM: Increased faculty presence on inpatient teaching services. Mayo Clin Proc. 2004, 79:332-336. 10.4065/79.3.332

11. Hanson JT, Pierce RG, Dhaliwal G: The new education frontier: clinical teaching at night . Acad Med. 


\section{Cureus}

2014, 89:215-218. 10.1097/ACM.0000000000000096

12. Weltz AS, Cimeno A, Kavic SM: Strategies for improving education on night-float rotations: a review . J Surg Educ. 2014, 72:297-301. 10.1016/j.jsurg.2014.09.002 